\section{Capacidade funcional e fatores associados em idosos do Sul do Brasil: um estudo de base populacional}

\author{
Functional status and associated factors among the \\ elderly in a southern Brazilian city: a population- \\ based study
}

\author{
${ }^{1}$ Universidade do Oeste de \\ Santa Catarina, Joaçaba, \\ Brasil. \\ 2 Programa de Pós- \\ Graduação em Saúde \\ Pública, Universidade \\ Federal de Santa Catarina, \\ Florianópolis, Brasil. \\ Correspondência \\ K. G. Peres \\ Programa de Pós-Graduação \\ em Saúde Pública, Centro \\ de Ciências da Saúde, \\ Universidade Federal de \\ Santa Catarina. \\ Rua Berlim 209, \\ Florianópolis, SC \\ 88037-325, Brasil. \\ karengp@ccs.ufsc.br
}

\begin{abstract}
The goal of this study was to estimate the prevalence of low functional capacity and associated factors in Joaçaba, Santa Catarina State, Brazil, in 2003-2004, using a household survey ( $n=345)$. Subjects who demonstrated difficulty in performing 6 or more activities or inability to conduct 3 of the activities according to the scale proposed by Rikli \& Jones were defined as having low functional capacity. A questionnaire covering socioeconomic, demographic, housing, and self-perceived socioeconomic variables was applied. The study included distribution of the target variables' simple frequency, chi-square association tests, and multiple logistic regression analysis. The response rate was $92.7 \%$, with a low functional prevalence of 37.1\% (95\% CI: 32.042.2). Mean age was 69.5 years. After adjusting for confounding variables, the factors associated with low functional capacity were: age $>70$ years, female gender, and negative self-perceived socioeconomic status. A high percentage of elderly citizens in Joaçaba show low functional capacity, leading to dependency. Health programs that delay the onset of such disabilities could contribute to healthier aging.
\end{abstract}

Aging; Frail Elderly; Health of the Elderly
Mariarosa Mendes Fiedler 1

Karen Glazer Peres 2

\section{Introdução}

O acelerado ritmo do crescimento da população idosa é observado mundialmente, inclusive no Brasil e em outros países latino-americanos 1,2. A expectativa de vida dos brasileiros que em 1900 não alcançava os 35 anos de idade, em 1950 atingiu 43 anos, em 2000, 68, com a expectativa de atingir os 80 anos em 2025 2,3.

O envelhecimento é um processo que provoca alterações e desgastes em vários sistemas funcionais, que ocorrem de forma progressiva e irreversível 4. O momento em que estas transformações ocorrem, quando passam a ser percebidas e como evoluem, diferencia-se de um indivíduo para o outro 5. Entretanto, em idades mais avançadas as limitações visuais, auditivas, motoras e intelectuais, bem como o surgimento de doenças crônico-degenerativas intensificamse, ocasionando a dependência nas atividades cotidianas. O resultante desses fatores é a diminuição da condição de saúde do idoso que acaba procurando com mais freqüência os serviços de saúde pública, principalmente aqueles vinculados ao Sistema Único de Saúde (SUS) 5,6,7.

Capacidade funcional pode ser definida como o potencial que os idosos apresentam para decidir e atuar em suas vidas de forma independente, no seu cotidiano ${ }^{8}$. As informações geradas pela avaliação da capacidade funcional possibilitam conhecer o perfil dos idosos usando-se ferramenta simples e útil, que pode auxiliar na 
definição de estratégias de promoção de saúde para os idosos, visando a retardar ou prevenir as incapacidades 9,10 .

A forma como o idoso vive, a qualidade dos últimos anos de sua vida e suas maiores limitações podem ser observadas por meio de estudos populacionais direcionados a esta faixa etária. No Brasil esses estudos ainda são raros, e os poucos que vêm sendo realizados refletem o perfil da população de grandes centros, envolvendo populações com costumes e características diferentes da população dos pequenos municípios como, por exemplo, a da Região Sul do Brasil 1,11.

O objetivo deste estudo foi realizar um inquérito populacional sobre a capacidade funcional dos idosos e conhecer os fatores associados no Município de Joaçaba, cidade de pequeno porte localizada no meio-oeste de Santa Catarina, Brasil.

\section{Método}

Foi realizado um estudo transversal com idosos, entre 60 e 94 anos de idade, residentes na zona urbana do Município de Joaçaba, entre o segundo semestre de 2003 e o primeiro de 2004.

Para cálculo do tamanho da amostra foram considerados os seguintes parâmetros: tamanho da população ( $\mathrm{N}=2.100)$ (http://www.datasus. gov.br, acessado em 14/Jul/2003), prevalência de capacidade funcional inadequada de $30 \%{ }^{1}$, erro igual a 5 pontos percentuais e intervalo de confiança de 95\% (IC95\%). O tamanho da amostra obtido ( $n=280$ ) foi corrigido em 1,2 devido ao efeito do desenho e acrescido de $10 \%$ devido a eventuais perdas e/ou recusas, totalizando 370 indivíduos. A seleção da amostra foi realizada pelo método de amostragem por conglomerados, a partir dos 23 setores censitários urbanos do município, segundo o Instituto Brasileiro de Geografia e Estatística (IBGE) 3. Os setores censitários foram considerados o primeiro estágio para o sorteio e os domicílios, o segundo estágio 12.

Foi aplicado um questionário estruturado na forma de entrevista para conhecer o nível da capacidade funcional e variáveis sócio-econômicas e demográficas (sexo, idade em anos, tipo de moradia - própria ou outra -, renda em salários mínimos, escolaridade em anos de estudo, profissão que exerceu - atividade leve ou pesada). Para avaliar a capacidade funcional foi utilizada uma escala de auto-avaliação com 12 tipos de atividades, proposta por Rikli \& Jones 13. Essa escala é composta pelas atividades: (a) tomar conta de suas necessidades pessoais como vestir-se; (b) tomar banho, usar o banheiro; (c) caminhar fora de casa (1 a 2 quarteirões); (d) fazer ativi- dades domésticas leves como cozinhar, tirar o pó, lavar pratos, varrer ou andar de um lado para o outro na casa; (e) subir ou descer escadas; (f) fazer compras no supermercado ou no centro; (g) levantar e carregar 5kg (como por exemplo um pacote de arroz); (h) caminhar 6 a 7 quarteirões; (i) caminhar 12 a 14 quarteirões; (j) levantar e carregar $13 \mathrm{~kg}$ de peso (como uma mala média grande); (k) fazer atividade doméstica pesada como aspirar, esfregar pisos, passar o rastelo; (l) fazer atividades vigorosas como andar grandes distâncias, cavoucar o jardim, mover objetos pesados, atividades de dança aeróbica ou ginástica vigorosa.

O instrumento utilizado para avaliar a capacidade funcional é de fácil aplicação e permite incluir todos os idosos, fisicamente dependentes e independentes, possibilitando, desta forma, uma estimativa da condição de interesse na população como um todo, da faixa etária estudada. As 12 atividades analisadas são categorizadas em: faço, faço com dificuldade ou ajuda e não posso fazer, recebendo escores iguais a 1, 2 e 3 , respectivamente, que, posteriormente são somadas. Para análise estatística, o desfecho "capacidade funcional" foi dicotomizado em adequada e inadequada. Foram consideradas com capacidade funcional inadequada aquelas pessoas com dificuldade para realizar seis ou mais atividades (categoria 2), ou ainda quando o indivíduo sentia-se inábil para fazer três atividades do total proposto (categoria 3).

A pesquisa foi realizada em nível domiciliar, com uma média de duas entrevistas por dia. A entrevista foi realizada pela autora do estudo e mais dois entrevistadores, acadêmicos do curso de Educação Física da Universidade do Oeste de Santa Catarina em Joaçaba. A equipe foi submetida a treinamento para a aplicação do questionário e realização da entrevista.

O programa estatístico utilizado foi o SPSS (SPSS Inc., Chicago, Estados Unidos). Para a análise estatística realizou-se a distribuição de freqüência simples das variáveis de interesse do estudo; testes de associação do qui-quadrado para verificar associação entre desfecho e variável exploratória e regressão logística múltipla para testar independência entre desfecho e variáveis associadas 14

Todas as variáveis que na análise bivariada apresentaram $\mathrm{p}<0,20$ foram selecionadas para o modelo final, como potenciais fatores de confusão. As variáveis foram introduzidas no modelo múltiplo conforme sua significância estatística, uma a uma, respeitando-se os blocos de variáveis: o das sócio-econômicas primeiro, seguido das demográficas, tipo de moradias e percepção da situação sócio-econômica. As variáveis per- 
maneceram no modelo múltiplo quando continuaram significativas $(\mathrm{p}<0,05)$ ou quando ajustaram o modelo 14.

Antes de iniciar a entrevista, foi entregue ao entrevistado um termo de consentimento, que considera os aspectos éticos presentes na Resolução no. 196/96, que normatiza a pesquisa em seres humanos. O projeto de investigação foi aprovado pelo Comitê de Ética em Pesquisa da Universidade de Passo Fundo (Passo Fundo, Rio Grande do Sul, Brasil).

\section{Resultados}

Do total da amostra, 345 (92,7\%) indivíduos participaram do estudo. Os idosos residentes na zona urbana do Município de Joaçaba caracterizam-se por ser a maioria mulher, morando em casa própria com seus familiares e considerando sua residência como boa ou muito boa. Apenas $26,1 \%$ da população de idosos apresentaram renda familiar igual a cinco salários mínimos ou mais, com percepção da sua situação econômica como ruim ou muito ruim relatada por $22,6 \%$ dos entrevistados. A escolaridade mais encontrada entre os participantes do estudo foi de três e quatro anos de estudos completos. Capacidade funcional inadequada foi observada em $37,1 \%$ (IC95\%: 28,7-45,7) dos idosos (Tabela 1).

A análise bivariada entre a prevalência de capacidade funcional e idade apresentou tendência de linearidade inversa $(p<0,001)$. A prevalência de capacidade funcional inadequada foi maior nas mulheres $(43,1 \%)$ que nos homens $(25,8 \%)$ $(p=0,002)$. Quanto menor a renda total do domicílio, maior a prevalência de capacidade funcional inadequada (Tabela 2).

Os que consideram sua situação econômica ruim ou muito ruim apresentaram-se associados à capacidade funcional inadequada quando comparados aos que a consideram boa ou muito boa ( $p=0,035$ ). Quanto ao nível de escolaridade percebeu-se que quanto menor o número de anos de estudos maior a proporção de indivíduos com capacidade funcional inadequada ( $p<0,001)$. Idosos cujas atividades profissionais caracterizaram-se como pesadas mostraram-se associados à capacidade funcional inadequada $(\mathrm{p}=0,036)$.

Após ajustamento para possíveis fatores de confusão, as seguintes variáveis permaneceram positivamente associadas à capacidade funcional inadequada: idade maior ou igual a 70 anos $(p<0,001)$, ser do sexo feminino $(p=0,002)$ e indivíduos que avaliaram sua situação econômica como razoável, ruim ou muito ruim $(\mathrm{p}=0,035)$ (Tabela 3).
Tabela 1

Distribuição da amostra de acordo com as variáveis dos idosos residentes na zona urbana de Joaçaba, Santa Catarina, Brasil, 2003/2004.

\begin{tabular}{|c|c|c|c|}
\hline Variáveis & $\mathrm{n}$ & $\%$ & IC95\% \\
\hline \multicolumn{4}{|l|}{ Idade (anos) } \\
\hline $60-69$ & 200 & 57,9 & $51,1-64,7$ \\
\hline 70-79 & 104 & 30,1 & $21,3-38,9$ \\
\hline $80 \mathrm{ou}+$ & 41 & 11,9 & $2,0-21,8$ \\
\hline \multicolumn{4}{|l|}{ Sexo } \\
\hline Masculino & 120 & 34,8 & $26,3-43,3$ \\
\hline Feminino & 225 & 65,2 & $59,0-71,4$ \\
\hline \multicolumn{4}{|c|}{ Renda total por residência (salário mínimo) * } \\
\hline$\geq 5$ & 90 & 26,1 & $17,0-35,2$ \\
\hline $2,1-4,9$ & 122 & 35,4 & $26,9-43,9$ \\
\hline $1,1-2$ & 112 & 32,5 & $23,8-41,2$ \\
\hline$<1$ & 21 & 6,1 & $0,0-16,3$ \\
\hline \multicolumn{4}{|l|}{ Anos de escolaridade (anos) } \\
\hline+8 & 44 & 12,8 & $2,9-22,7$ \\
\hline $5-8$ & 42 & 12,2 & $2,3-22,1$ \\
\hline $3-4$ & 167 & 48,4 & $40,8-56,0$ \\
\hline 2 & 27 & 7,8 & $0,0-18,0$ \\
\hline 1 & 31 & 9,0 & $0,0-19,1$ \\
\hline Não cursou & 34 & 9,9 & $0,0-19,9$ \\
\hline \multicolumn{4}{|l|}{ Moradia } \\
\hline Própria & 301 & 87,2 & $83,4-91,0$ \\
\hline Outra & 44 & 12,8 & $2,9-22,7$ \\
\hline \multicolumn{4}{|l|}{ Com quem mora } \\
\hline Mora só & 62 & 18,0 & $8,4-27,6$ \\
\hline Com esposo(a) & 123 & 35,7 & $27,2-44,2$ \\
\hline Com filhos(as) & 75 & 21,7 & $12,4-31,1$ \\
\hline Com esposo(a) + filho(as) & 66 & 19,1 & $9,6-28,6$ \\
\hline Outros & 19 & 5,5 & $0,0-15,7$ \\
\hline \multicolumn{4}{|l|}{ Como considera sua residência } \\
\hline Boa/Muito boa & 248 & 72,0 & $64,4-77,6$ \\
\hline Razoável & 80 & 23,2 & $13,9-32,4$ \\
\hline Ruim/Muito ruim & 17 & 4,9 & $0,0-15,2$ \\
\hline \multicolumn{4}{|c|}{ Como avalia sua situação econômica } \\
\hline Muito boa/Boa & 97 & 28,1 & $19,1-37,0$ \\
\hline Razoável & 170 & 49,3 & $41,8-56,8$ \\
\hline Ruim/Muito ruim & 78 & 22,6 & $12,8-31,9$ \\
\hline \multicolumn{4}{|l|}{ Profissão que mais exerceu } \\
\hline Atividade leve & 94 & 27,2 & $18,2-36,2$ \\
\hline Atividade pesada & 251 & 72,8 & $67,3-78,3$ \\
\hline \multicolumn{4}{|l|}{ Capacidade funcional } \\
\hline Adequada & 217 & 62,9 & $56,5-69,3$ \\
\hline Inadequada & 128 & 37,1 & $28,7-45,7$ \\
\hline
\end{tabular}

* Salário mínimo do período: $\mathrm{R} \$ 240,00$. 
Tabela 2

Análise bivariada de inadequada capacidade funcional de acordo com as variáveis sóciodemográficas, condições de moradia e autopercepção dos idosos residentes na zona urbana de Joaçaba, Santa Catarina, Brasil, 2003/2004.

\begin{tabular}{lccc}
\hline Variáveis & $\begin{array}{c}\text { Inadequada } \\
\text { capacidade (\%) }\end{array}$ & IC95\% \\
\hline
\end{tabular}

\begin{tabular}{|c|c|c|c|}
\hline \multicolumn{4}{|l|}{ Idade (anos) } \\
\hline $60-69$ & 22,5 & \multirow[t]{3}{*}{$<0,001$} & $16,7-28,3$ \\
\hline $70-79$ & 51,9 & & $35,3-68,3$ \\
\hline $80 \mathrm{ou}+$ & 70,7 & & $56,8-84,6$ \\
\hline \multicolumn{4}{|l|}{ Sexo } \\
\hline Masculino & 25,8 & \multirow[t]{2}{*}{0,002} & $18,0-33,6$ \\
\hline Feminino & 43,1 & & $36,6-49,6$ \\
\hline \multicolumn{4}{|c|}{ Renda total por residência (salário mínimo) ** } \\
\hline$\geq 5$ & 33,3 & \multirow[t]{4}{*}{0,098} & $23,6-43,0$ \\
\hline $2,1-4,9$ & 36,1 & & $27,6-44,6$ \\
\hline $1,1-2$ & 36,6 & & $27,7-45,5$ \\
\hline$<1$ & 61,9 & & $41,1-82,7$ \\
\hline \multicolumn{4}{|l|}{ Anos de escolaridade (anos) } \\
\hline+8 & 18,2 & \multirow[t]{6}{*}{$<0,001$} & $6,8-29,6$ \\
\hline $5-8$ & 28,6 & & $14,9-42,3$ \\
\hline $3-4$ & 37,7 & & $30,3-45,0$ \\
\hline 2 & 37,0 & & $18,8-55,2$ \\
\hline 1 & 45,2 & & $27,7-62,7$ \\
\hline Não cursou & 61,8 & & $45,5-78,1$ \\
\hline \multicolumn{4}{|l|}{ Moradia } \\
\hline Própria & 36,2 & \multirow[t]{2}{*}{0,467} & $30,8-41,6$ \\
\hline Outra & 43,2 & & $28,6-48,4$ \\
\hline \multicolumn{4}{|l|}{ Com quem mora } \\
\hline Mora só & 38,7 & \multirow[t]{5}{*}{0,996} & $26,6-50,8$ \\
\hline Com esposo(a) & 30,9 & & $22,7-39,1$ \\
\hline Com filhos(as) & 53,3 & & $42,0-64,6$ \\
\hline Com esposo(a) + filho(as) & 28,8 & & $17,9-39,7$ \\
\hline Outros & 36,8 & & $25,7-47,9$ \\
\hline \multicolumn{4}{|l|}{ Como considera sua residência } \\
\hline Boa/Muito boa & 30,3 & \multirow[t]{3}{*}{0,651} & $24,6-36,0$ \\
\hline Razoável & 32,5 & & $22,2-42,8$ \\
\hline Ruim/Muito ruim & 41,2 & & $17,8-64,6$ \\
\hline \multicolumn{4}{|c|}{ Como avalia sua situação econômica } \\
\hline Muito boa/Boa & 26,8 & \multirow[t]{3}{*}{0,012} & $18,0-35,6$ \\
\hline Razoável & 39,4 & & $32,0-46,7$ \\
\hline Ruim/Muito ruim & 44,9 & & $33,9-55,9$ \\
\hline \multicolumn{4}{|l|}{ Profissão que mais exerceu } \\
\hline Atividade leve & 27,7 & \multirow[t]{2}{*}{0,036} & $18,7-36,7$ \\
\hline Atividade pesada & 40,6 & & $34,5-46,9$ \\
\hline
\end{tabular}

* p do qui-quadrado (correção de Yates).

** Salário mínimo do período: R\$240,00.

\section{Discussão}

A boa taxa de resposta obtida (92,7\%) contribuiu para a validade interna da pesquisa. Os idosos entrevistados foram comparados com a população residente no município segundo faixa etária e sexo, constatando-se que as proporções encontradas foram muito semelhantes. Tal observação reduz possível viés de seleção e contribui para a validade externa da pesquisa. Entretanto, ao extrapolar os resultados para a população idosa em geral, recomenda-se considerar as possíveis diferenças culturais, sócio-econômicas e estilo de vida. Conforme o observado neste e em outros estudos, esses fatores podem estar associados à prevalência da capacidade funcional inadequada.

Neste estudo, observou-se que $37,1 \%$ dos idosos apresentaram capacidade funcional inadequada. Inquérito domiciliar realizado no Nordeste mostrou que pouco mais da metade dos idosos $(52,3 \%)$ apresentaram autonomia total para a realização das atividades da vida diária e $35 \%$ referiram necessitar de ajuda para realizar até três atividades 15. Em São Paulo, estudo realizado em amostra representativa do município, $53 \%$ dos idosos ( $\geq 60$ anos) referiram autonomia total nas atividades da vida diária, enquanto $29 \%$ dos entrevistados necessitavam de ajuda parcial ou total para realizar até três atividades 1 . Estudo realizado na periferia de Goiânia $(n=107)$, com idosos atendidos por uma equipe de saúde da família mostrou que 40,9\% destes eram dependentes na realização das atividades da vida diária 16. Embora a prevalência de capacidade funcional inadequada encontrada no nosso estudo tenha sido semelhante à de outros estudos nacionais, não encontrou-se outro estudo nacional que tenha utilizado o mesmo protocolo para avaliar o referido desfecho devendo, portanto, considerar esta limitação ao serem feitas comparações diretas entre os resultados dos diferentes estudos.

A idade mostrou-se um fator fortemente associado à perda da capacidade funcional. $\mathrm{Na}$ nossa pesquisa, os idosos com 70 anos ou mais tiveram maior chance de ter capacidade funcional inadequada, quando comparados àqueles com entre 60 e 69 anos, corroborando os resultados encontrados em outros estudos 10,17.

Em estudo realizado em 13 distritos de Portugal ( $\mathrm{n}=1.354$ ) com indivíduos com 75 anos ou mais, foi encontrada prevalência de capacidade funcional inadequada menor do que a maioria dos estudos brasileiros, apesar da idade média dos integrantes da amostra ter sido maior. Adicionalmente, somente $3,2 \%$ da amostra foram considerados dependentes e $4,3 \%$ foram considerados quase dependentes. No mesmo estudo, observou-se que no grupo autônomo predomi- 
Distribuição das variáveis associadas com inadequada capacidade funcional após ajuste por idade, sexo e escolaridade dos idosos residentes na zona urbana de Joaçaba, Santa Catarina, Brasil, 2003/2004.

\begin{tabular}{|c|c|c|c|c|}
\hline Variáveis & OR (IC95\%) & p (bruto) & OR (IC95\%) & p (ajustado) \\
\hline Renda total por residência (salários mínimos) & & 0,058 & & * \\
\hline$\geq 5$ & 1,0 & & * & \\
\hline $2-4$ & $1,1(0,7-1,9)$ & & * & \\
\hline$<2$ & $3,3(1,2-8,7)$ & & * & \\
\hline Escolaridade (anos) & & 0,007 & & * \\
\hline+8 & 1,0 & & * & \\
\hline $5-8$ & $1,8(0,7-5,0)$ & & * & \\
\hline $0-4$ & $3,2(1,4-7,2)$ & & * & \\
\hline Sexo & & 0,002 & & 0,007 \\
\hline Masculino & 1,0 & & 1,0 & \\
\hline Feminino & $2,2(1,3-3,5)$ & & $2,1(1,2-3,6)$ & \\
\hline Idade (anos) & & $<0,001$ & & \\
\hline $60-69$ & 1,0 & & 1,0 & $<0,001$ \\
\hline $70-79$ & $3,7(2,2-6,2)$ & & $4,2(2,5-7,2)$ & \\
\hline $80 \mathrm{ou}+$ & $8,3(3,9-17,7)$ & & $8,6(4,0-18,8)$ & \\
\hline Como avalia sua situação econômica & & 0,035 & & 0,018 \\
\hline Muito boa/Boa & 1,0 & & 1,0 & \\
\hline Razoável & $1,8(1,3-3,1)$ & & $2,1(1,1-3,8)$ & \\
\hline Ruim/Muito ruim & $2,2(1,2-4,2)$ & & $3,4(1,7-7,0)$ & \\
\hline
\end{tabular}

* Excluído após ajuste com outras variáveis.

navam os homens e mais indivíduos pertencentes à menor faixa etária. Ser do sexo feminino e mais velho significou ter menor autonomia 17 .

O Estudo Longitudinal Americano de Envelhecimento 10 com indivíduos com 70 anos de idade ou mais ( $\mathrm{n}=7.527$ ), analisou as mudanças do estado funcional utilizando um índice composto por atividades da vida diária e atividades instrumentais da vida diária. Nesse estudo, além do declínio funcional estar positivamente associado com o aumento da idade, destacou-se, também, a diminuição da qualidade auditiva e visual, com ocorrências de tonturas e outras instabilidades, levando à maior dependência 2 .

A prevalência de capacidade funcional inadequada encontrada em Joaçaba foi maior nas mulheres que nos homens. As mulheres, que são maioria em grupos de idosos, apesar de ter maior longevidade, apresentam maiores limitações ou maior perda da capacidade funcional, corroborando os estudos de Brill et al. 18 nos Estados Unidos, Souza et al. 17 em Portugal e Feliciano et al. 19 em São Carlos, São Paulo. Embora esse fato tenha sido observado em outros estudos, em nenhum deles houve uma explicação para essa perda da capacidade funcional mais acentuada no sexo feminino.
Fabrício et al. ${ }^{20}$, ao analisar os resultados de estudos de causas e conseqüências de quedas em idosos residentes em Ribeirão Preto, São Paulo, observou uma freqüência de quedas maior em mulheres do que em homens, citando como conseqüências destas quedas a redução na capacidade funcional. No mesmo estudo os autores observam a ocorrência de fraturas como resultado agravante da queda, sugerindo uma relação desta condição com a presença de osteoporose prevalente no sexo feminino 20 . As mulheres têm maior perda de massa muscular e massa óssea com o envelhecimento, caracterizando-se como potenciais fatores responsáveis pela diminuição da capacidade funcional, que devem ser explorados em futuros estudos 20 .

A atividade física regular vem sendo considerada como forma de manutenção da aptidão física em indivíduos idosos, citada na literatura como forma de atenuar e reverter a perda de massa muscular, contribuindo para preservar a autonomia funcional e o envelhecimento saudável 20,21.

A percepção que o idoso tem de sua situação econômica, esteve mais associada à capacidade funcional inadequada do que a renda domiciliar. Estudos sobre auto-avaliação ou autopercepção 
têm sido bastante relatados na literatura 10,11, sugerindo que o idoso percebe sua saúde conforme as possibilidades que tem para enfrentar seus problemas. A forma como o idoso percebe sua vida, suas expectativas e suas preocupações podem influenciar sua saúde e sua autonomia.

Dos fatores que estiveram associados a essa diminuição na capacidade funcional alguns podem ser revertidos ou minimizados. A percepção que as pessoas têm de sua situação econômica, a falta de segurança que sentem em relação ao pequeno poder aquisitivo de que dispõem, aumentam as chances de perda de autonomia. Esse fator pode estar ligado também à forma como o idoso sente-se no momento, isto é, após a aposentadoria alguns idosos passam a ter de viver com rendas menores, e este fator pode influenciar sua auto-estima.

Os dados encontrados neste estudo sugerem que a população idosa que vive em Joaçaba, um município de pequeno porte no Sul do Brasil,

\section{Resumo}

Estimar a prevalência de capacidade funcional inadequada e fatores associados na população idosa de Joaçaba, Santa Catarina, Brasil, entre 2003 e 2004. Realizou-se um inquérito populacional domiciliar ( $n=$ 345). Foram considerados com capacidade funcional inadequada indivíduos com dificuldade na realização de seis atividades, ou inabilidade para realizar três da escala proposta por Rikli \& Jones. Foi aplicado um questionário contendo questões sobre variáveis sócio-econômicas, demográficas, tipo de moradia e percepção da própria situação econômica. Realizou-se a distribuição de freqüência simples das variáveis de interesse, teste de associação do qui-quadrado e análise de regressão logística múltipla não condicional. A taxa de resposta foi de 92,7\%, prevalência de capacidade funcional inadequada de 37,1\% (IC95\%: 32,0-42,2). A idade média foi de 69,5 anos. Após ajustamento para fatores de confusão, permaneceram associadas à capacidade funcional inadequada ter 70 anos ou mais, ser do sexo feminino e apresentar autopercepção negativa de sua situação econômica. Muitos dos idosos de Joaçaba apresentam capacidade funcional inadequada, levando à dependência. Programas de saúde que retardem o aparecimento de incapacidades, bem como a realização de atividades sociais poderiam contribuir para um envelhecimento saudável.

Envelhecimento; Idoso Débil; Saúde do Idoso tem sido acometida de limitações no seu cotidiano, convivendo com dependência na realização de atividades simples. Os resultados reforçam a importância de avaliar a capacidade funcional do idoso e transformar este fator como parte integrante da avaliação clínica. A possibilidade de intervir na promoção de saúde com ações que retardem o aparecimento das incapacidades, e viabilizar a reabilitação quando estas forem detectadas, pode reduzir o número de idosos dependentes, melhorando a qualidade de vida dos brasileiros. Políticas públicas de saúde voltadas para programas específicos de prevenção a fatores relacionados à incapacidade funcional como, por exemplo, hipertensão arterial, audição, visão, entre outros, poderiam contribuir na qualidade de vida desta população. Adicionalmente, atividades sociais e culturais que fortaleçam grupos de idosos poderiam ser desenvolvidas nos espaços coletivos existentes e, também, junto às instituições de ensino superior do município.

\section{Colaboradores}

M. M. Fiedler escolheu o tema, coletou os dados, construiu o banco de dados e elaborou a discussão dos resultados. K. G. Peres participou de todas as etapas acima citadas, realizou a análise estatística, colaborou na discussão, organização e revisão crítica da versão final do manuscrito. 


\section{Referências}

1. Ramos LR, Rosa TEC, Oliveira ZM, Medina MCG, Santos FRG. Perfil do idoso em área metropolitana na região sudeste do Brasil: resultados de inquérito domiciliar. Rev Saúde Pública 1993; 27:87-94.

2. Pacheco RO, Santos SSC. Avaliação global de idosos em unidades de PSF. Textos sobre Envelhecimento 2004; 7(2).

3. Instituto Brasileiro de Geografia e Estatística. Censo demográfico 2000: características da população. http://www.ibge.gov.br/ (acessado em 16/ $\mathrm{Jul} / 2003)$.

4. Caromano FA, Jung TC. Estudo comparativo do desempenho em testes de força muscular entre indivíduos jovens e idosos através da miometria. Revista de Fisioterapia 1999; 6:101-12.

5. Naranjo JLR, Estrada LC, Ferra RR, Jiménez IP, Rivero JLP. Autonomía e validismo en la tercera edad. Rev Cubana Med Gen Integr 2001; 17:22-6.

6. Ramos LR. Fatores determinantes do envelhecimento saudável em idosos residentes em centro urbano: Projeto Epidoso, São Paulo. Cad Saúde Pública 2003; 19:793-7.

7. Sanglard RCF, Henriques GRP, Ribeiro ASB, Corrêa AL, Pereira JS. Alterações dos parâmetros da marcha em função das queixas de instabilidade postural e quedas em idosos. Fitness \& Performance Journal 2004; 3:149-56.

8. Matsudo SM. Avaliação do idoso: física e funcional. Londrina: Midiograf; 2000.

9. Xavier F, Ferraz MPT, Bisol LW, Fernandes DD, Schwanke C, Moringuchi EH. Octagenários de Veranópolis: as condições psicológicas, sociais e de saúde geral de um grupo representativo de idosos com mais de 80 anos residentes na comunidade. Rev AMRIGS 2000; 44:25-9.

10. Lee Y. The predictive value of self assessed general, physical, and mental health on functional decline and mortality in older adults. J Epidemiol Community Health 2000; 54:123-9.
11. Lima-Costa MF, Firmo JOA, Uchôa E. Estrutura da auto-avalição da saúde em idosos: Projeto Bambuí. Rev Saúde Pública 2004; 38:827-34.

12. Barros FC, Victora CG. Epidemiologia da saúde infantil: um manual para diagnósticos comunitários. São Paulo: Editora Hucitec; 1998.

13. Rikli RE, Jones CJ. Development and validation of a functional fitness test for community-residing older adults. J Aging Phys Act 1999; 7:129-61.

14. Hosmer DW, Lemeshow S. Applied logistic regression. New York: John Wiley and Sons; 1989.

15. Coelho Filho JM, Ramos RL. Epidemiologia do envelhecimento no Nordeste do Brasil: resultados de inquérito domiciliar. Rev Saúde Pública 1999; 33:445-53.

16. Nakatani AYK, Costa EFA, Teles SA, Silva LB, Rêgo MAB, Silva-e-Souza AC, et al. Perfil sócio-demográfico e avaliação funcional de idosos atendidos por uma equipe de saúde da família na periferia de Goiânia, Goiás. Rev Soc Bras Clín Méd 2003; 1:131-6.

17. Souza L, Galante H, Figueredo D. Qualidade de vida e bem-estar dos idosos: um estudo exploratório na população portuguesa. Rev Saúde Pública 2003; 37:364-71.

18. Brill PA, Macera CA, Davis SNB, Gordon N. Muscular strength and physical function. Med Sci Sport Exerc 2000; 32:412-6.

19. Feliciano AB, Moraes AS, Freitas ICM. O perfil dos idosos de baixa renda no Município de São Carlos, São Paulo, Brasil: um estudo epidemiológico. Cad Saúde Pública 2004; 20:1575-85.

20. Fabrício SCC, Rodrigues RAP, Costa Jr. ML. Causas e conseqüências de quedas de idosos atendidos em hospital público. Rev Saúde Pública 2004; 38:93-9.

21. Sheppard RJ. Envelhecimento, atividade física e saúde. São Paulo: Phorte Editora; 2003.

Recebido em 20/Out/2006

Versão final reapresentada em 16/Jul/2007

Aprovado em 30/Jul/2007 